\title{
Existence results for fractional integral inclusions via nonlinear alternative for contractive maps
}

\author{
Ahmed Alsaedi ${ }^{1}$, Sotiris K Ntouyas ${ }^{2}$, Bashir Ahmad ${ }^{1 *}$ and Hamed H Alsulami ${ }^{1}$
}

\section{"Correspondence:}

bashirahmad_qau@yahoo.com

'Department of Mathematics,

Faculty of Science, King Abdulaziz

University, P.O. Box 80203, Jeddah,

21589, Saudi Arabia

Full list of author information is

available at the end of the article

\begin{abstract}
In this paper, a new existence result is obtained for a fractional multivalued problem with fractional integral boundary conditions by applying a (Krasnoselskii type) fixed-point result for multivalued maps due to Petryshyn and Fitzpatric [Trans. Am. Math. Soc. 194:1-25, 1974]. The case for lower semi-continuous multivalued maps is also discussed. An example for the illustration of our main result is presented.
\end{abstract}

MSC: 34A60; 34A08

Keywords: fractional differential inclusions; nonlocal boundary conditions; fixed-point theorems

\section{Introduction}

The theory of fractional differential equations and inclusions has developed into an important field of investigation due to its extensive applications in numerous branches of physics, economics, and engineering sciences [1-4]. The nonlocal behavior exhibited by a fractional-order differential operator makes it distinct from the integer-order differential operator. It means that the future state of a dynamical system or process involving fractional derivatives depends on its current state as well its past states. In fact, differential equations of arbitrary order are capable of describing memory and hereditary properties of several materials and processes. This characteristic of fractional calculus has contributed to its popularity and has convinced many researchers of the need to shift their focus from classical integer-order models to fractional-order models. There has been a great surge in developing new theoretical aspects such as periodicity, asymptotic behavior, and numerical methods for fractional equations. For some recent work on the topic, see [5-18] and the references cited therein.

In this paper, we consider the following boundary value problem of fractional differential inclusions with fractional integral boundary conditions:

$$
\left\{\begin{array}{l}
-D^{\alpha} x(t) \in A F(t, x(t))+B I^{\beta} G(t, x(t)), \quad 2<\alpha \leq 3, t \in[0,1], \\
D^{\delta} x(0)=D^{\delta+1} x(0)=0, \quad D^{\delta} x(1)=\int_{0}^{\eta} D^{\delta} x(s) d s, \quad 0<\eta<1,
\end{array}\right.
$$

where $0<\delta \leq 1,2<\alpha-\delta<3, \beta>0, D^{(\cdot)}$ denotes the Riemann-Liouville fractional derivative of order $(\cdot), F, G:[0,1] \times \mathbb{R} \rightarrow \mathcal{P}(\mathbb{R})$ are multivalued maps, $\mathcal{P}(\mathbb{R})$ is the family of all nonempty subsets of $\mathbb{R}$ and $A, B$ are real constants.

(02014 Alsaedi et al.; licensee Springer. This is an Open Access article distributed under the terms of the Creative Commons Attribution License (http://creativecommons.org/licenses/by/2.0), which permits unrestricted use, distribution, and reproduction in any medium, provided the original work is properly cited. 
We establish two new existence results for the problem (1.1). The first result relies on a nonlinear alternative for contractive maps, while in the second result, we shall combine the nonlinear alternative of Leray-Schauder type for single-valued maps with a selection theorem due to Bressan and Colombo for lower semi-continuous multivalued maps with nonempty closed and decomposable values.

The paper is organized as follows. In Section 2, we recall some preliminary facts that we need in the sequel and Section 3 deals with the main results.

\section{Preliminaries}

Let us recall some basic definitions of the fractional calculus [1-3].

Definition 2.1 The Riemann-Liouville derivative of fractional order $q$ for a continuous function $g:(0, \infty) \rightarrow \mathbb{R}$ is defined as

$$
D_{0+}^{q} g(t)=\frac{1}{\Gamma(n-q)}\left(\frac{d}{d t}\right)^{n} \int_{0}^{t}(t-s)^{n-q-1} g(s) d s, \quad n-1<q<n, n=[q]+1,
$$

where $[q]$ denotes the integer part of the real number $q$.

Definition 2.2 The Riemann-Liouville fractional integral of order $q$ for a function $g$ : $(0, \infty) \rightarrow \mathbb{R}$ is defined as

$$
I^{q} g(t)=\frac{1}{\Gamma(q)} \int_{0}^{t} \frac{g(s)}{(t-s)^{1-q}} d s, \quad q>0
$$

provided the integral exists.

Observe that the substitution $x(t)=I^{\delta} y(t)=D^{-\delta} y(t)$ transforms the problem (1.1) to the following form:

$$
\left\{\begin{array}{l}
-D^{\alpha-\delta} y(t) \in A F\left(t, I^{\delta} y(t)\right)+B I^{\beta} G\left(t, I^{\delta} y(t)\right), \quad t \in[0,1] \\
y(0)=0, \quad y^{\prime}(0)=0, \quad y(1)=\int_{0}^{\eta} y(s) d s
\end{array}\right.
$$

To define the solutions of the problem (1.1), we need the following lemma. Though the proof of this lemma involves standard arguments, we trace its proof for the convenience of the reader.

Lemma 2.3 For any $h \in C(0,1) \cap L(0,1)$, the unique solution of the linear fractional boundary value problem

$$
\left\{\begin{array}{l}
-D^{\alpha-\delta} y(t)=h(t), \quad t \in[0,1], \\
y(0)=y^{\prime}(0)=0, \quad y(1)=\int_{0}^{\eta} y(s) d s, \quad 0<\eta<1,
\end{array}\right.
$$

is

$$
y(t)=-I^{\alpha-\delta} h(t)+\frac{(\alpha-\delta) t^{\alpha-\delta-1}}{\alpha-\delta-\eta^{\alpha-\delta}}\left(I^{\alpha-\delta} h(1)-I^{\alpha-\delta+1} h(\eta)\right) .
$$


Proof It is well known [3] that the solution of fractional differential equation in (2.2) can be written as

$$
y(t)=-I^{\alpha-\delta} h(t)+c_{1} t^{\alpha-\delta-1}+c_{2} t^{\alpha-\delta-2}+c_{3} t^{\alpha-\delta-3},
$$

where $c_{1}, c_{2}, c_{3} \in \mathbb{R}$ are arbitrary constants. Using the boundary conditions in (2.2), we find that $c_{2}=0, c_{3}=0$, and

$$
c_{1}=\frac{\alpha-\delta}{\alpha-\delta-\eta^{\alpha-\delta}}\left(I^{\alpha-\delta} h(1)-I^{\alpha-\delta+1} h(\eta)\right) .
$$

Substituting these values in (2.3) yields

$$
y(t)=-I^{\alpha-\delta} h(t)+\frac{(\alpha-\delta) t^{\alpha-\delta-1}}{\alpha-\delta-\eta^{\alpha-\delta}}\left(I^{\alpha-\delta} h(1)-I^{\alpha-\delta+1} h(\eta)\right) .
$$

Notice that $\alpha \neq \delta+\eta^{\alpha-\delta}$ in view of the given values of the parameters involved in the expression. This completes the proof.

Thus, the solution of the equation $-D^{\alpha} x(t)=h(t)$ subject to the boundary conditions given by (1.1) can be written as

$$
\begin{aligned}
x(t)= & I^{\delta} y(t) \\
= & I^{\delta}\left[-I^{\alpha-\delta} h(t)+\frac{(\alpha-\delta) t^{\alpha-\delta-1}}{\alpha-\delta-\eta^{\alpha-\delta}}\left(I^{\alpha-\delta} h(1)-I^{\alpha-\delta+1} h(\eta)\right)\right] \\
= & -I^{\alpha} h(t)+\frac{(\alpha-\delta)}{\alpha-\delta-\eta^{\alpha-\delta}}\left(I^{\alpha-\delta} h(1)-I^{\alpha-\delta+1} h(\eta)\right) \int_{0}^{t} \frac{(t-s)^{\delta-1}}{\Gamma(\delta)} s^{\alpha-\delta-1} d s \\
= & -I^{\alpha} h(t)+\frac{(\alpha-\delta)}{\alpha-\delta-\eta^{\alpha-\delta}}\left(I^{\alpha-\delta} h(1)-I^{\alpha-\delta+1} h(\eta)\right) \\
& \times\left\{\frac{t^{\alpha-1}}{\Gamma(\delta)} \int_{0}^{1}(1-v)^{\delta-1} v^{\alpha-\delta-1} d v\right\},
\end{aligned}
$$

where we have used the substitution $s=v t$ in the integral of the last term. Using the relation for the Beta function $B(\cdot, \cdot)$,

$$
B(\beta+1, \alpha)=\int_{0}^{1}(1-u)^{\alpha-1} u^{\beta} d u=\frac{\Gamma(\alpha) \Gamma(\beta+1)}{\Gamma(\alpha+\beta+1)},
$$

we find that

$$
\begin{aligned}
x(t) & =-I^{\alpha} h(t)+\frac{(\alpha-\delta)}{\alpha-\delta-\eta^{\alpha-\delta}}\left(I^{\alpha-\delta} h(1)-I^{\alpha-\delta+1} h(\eta)\right)\left\{\frac{t^{\alpha-1}}{\Gamma(\delta)}\left(\frac{\Gamma(\delta) \Gamma(\alpha-\delta)}{\Gamma(\alpha)}\right)\right\} \\
& =-I^{\alpha} h(t)+\frac{\Gamma(\alpha-\delta+1) t^{\alpha-1}}{\left(\alpha-\delta-\eta^{\alpha-\delta}\right) \Gamma(\alpha)}\left(I^{\alpha-\delta} h(1)-I^{\alpha-\delta+1} h(\eta)\right) .
\end{aligned}
$$

Let $\mathcal{C}=C([0,1], \mathbb{R})$ denote the Banach space of all continuous functions from $[0,1] \rightarrow \mathbb{R}$ endowed with the norm defined by $\|x\|=\sup \{|x(t)|, t \in[0,1]\}$.

To establish the main results of this paper, we use the following form of the nonlinear alternative for contractive maps [19, Corollary 3.8]. 
Theorem 2.4 Let $X$ be a Banach space, and $D$ a bounded neighborhood of $0 \in X$. Let $H_{1}: X \rightarrow \mathcal{P}_{c p, c}(X)$ (here $\mathcal{P}_{c p, c}(X)$ denotes the family of all nonempty, compact and convex subsets of $X)$ and $H_{2}: \bar{D} \rightarrow \mathcal{P}_{c p, c}(X)$ two multivalued operators satisfying

(a) $H_{1}$ is contraction, and

(b) $\mathrm{H}_{2}$ is upper semi-continuous (u.s.c. for shortly) and compact.

Then, if $\mathrm{H}=\mathrm{H}_{1}+\mathrm{H}_{2}$, either

(i) H has a fixed point in $\bar{D}$ or

(ii) there is a point $u \in \partial D$ and $\lambda \in(0,1)$ with $u \in \lambda H(u)$.

Definition 2.5 A multivalued map $F:[0,1] \times \mathbb{R} \rightarrow \mathcal{P}_{c p, c}(\mathbb{R})$ is said to be $L^{1}$-Carathéodory if

(i) $t \rightarrow F(t, x)$ is measurable for each $x \in \mathbb{R}$,

(ii) $x \rightarrow F(t, x)$ is upper semi-continuous for almost all $t \in[0,1]$, and

(iii) for each real number $\rho>0$, there exists a function $h_{\rho} \in L^{1}\left([0,1], \mathbb{R}^{+}\right)$such that

$$
\|F(t, u)\|:=\sup \{|v|: v \in F(t, u)\} \leq h_{\rho}(t), \quad \text { a.e. } t \in[0,1]
$$

for all $u \in \mathbb{R}$ with $\|u\| \leq \rho$.

Denote

$$
S_{F, x}=\left\{v \in L^{1}([0,1], \mathbb{R}): v(t) \in F(t, x(t)) \text { a.e. } t \in[0,1]\right\} .
$$

Lemma 2.6 (Lasota and Opial [20]) Let X be a Banach space. Let $F:[0,1] \times \mathbb{R} \rightarrow \mathcal{P}_{c p, c}(\mathbb{R})$ be an $L^{1}$ Carathéodory multivalued map and let $\Theta$ be a linear continuous mapping from $L^{1}([0,1], \mathbb{R})$ to $C([0,1], X)$. Then the operator

$$
\Theta \circ S_{F}: C([0,1], \mathbb{R}) \rightarrow \mathcal{P}_{c p, c}(C([0,1], \mathbb{R})), \quad x \mapsto\left(\Theta \circ S_{F}\right)(x)=\Theta\left(S_{F, x}\right)
$$

is a closed graph operator in $C([0,1], \mathbb{R}) \times C([0,1], \mathbb{R})$.

\section{Existence results}

Before presenting the main results, we define the solutions of the boundary value problem (1.1).

Definition 3.1 A function $x \in A C^{2}([0,1], \mathbb{R})$ is said to be a solution of the problem (1.1) if $D^{\delta} x(0)=D^{\delta+1} x(0)=0, D^{\delta} x(1)=\int_{0}^{\eta} D^{\delta} x(s) d s$ and there exist functions $f \in S_{F, x}, g \in S_{G, x}$ such that

$$
\begin{aligned}
x(t)= & -A \int_{0}^{t} \frac{(t-s)^{\alpha-1}}{\Gamma(\alpha)} f(s) d s-B \int_{0}^{t} \frac{(t-s)^{\alpha+\beta-1}}{\Gamma(\alpha+\beta)} g(s) d s \\
& +Q t^{\alpha-1}\left[A \int_{0}^{1} \frac{(1-s)^{\alpha-\delta-1}}{\Gamma(\alpha-\delta)} f(s) d s+B \int_{0}^{1} \frac{(1-s)^{\alpha-\delta+\beta-1}}{\Gamma(\alpha-\delta+\beta)} g(s) d s\right. \\
& \left.-A \int_{0}^{\eta} \frac{(\eta-s)^{\alpha-\delta}}{\Gamma(\alpha-\delta+1)} f(s) d s-B \int_{0}^{\eta} \frac{(\eta-s)^{\alpha-\delta+\beta}}{\Gamma(\alpha-\delta+\beta+1)} g(s) d s\right],
\end{aligned}
$$


where

$$
Q=\frac{\Gamma(\alpha-\delta+1)}{\left(\alpha-\delta-\eta^{\alpha-\delta}\right) \Gamma(\alpha)} .
$$

In the sequel, we set

$$
\begin{aligned}
& Z_{1}=\frac{|A|}{\Gamma(\alpha+1)}+\frac{|A||Q|}{\Gamma(\alpha-\delta+1)}+\frac{|A||Q| \eta^{\alpha-\delta+1}}{\Gamma(\alpha-\delta+2)}, \\
& Z_{2}=\frac{|B|}{\Gamma(\alpha+\beta+1)}+\frac{|B||Q|}{\Gamma(\alpha-\delta+\beta+1)}+\frac{|B||Q| \eta^{\alpha-\delta+\beta+1}}{\Gamma(\alpha-\delta+\beta+2)} .
\end{aligned}
$$

\section{Theorem 3.2 Assume that}

$\left(\mathrm{H}_{1}\right) F:[0,1] \times \mathbb{R} \rightarrow \mathcal{P}_{c p, c}(\mathbb{R})$ is an $L^{1}$ Carathéodory multivalued map;

$\left(\mathrm{H}_{2}\right)$ there exists a function $k \in C\left([0,1], \mathbb{R}^{+}\right)$such that

$$
H(F(t, x), F(t, y)) \leq k(t)\|x-y\| \quad \text { a.e. } t \in[0,1]
$$

for all $x, y \in C([0,1], \mathbb{R})$ and $Z_{1}\|k\|<1$, where $Z_{1}$ is given by (3.2);

$\left(\mathrm{H}_{3}\right) \quad G:[0,1] \times \mathbb{R} \rightarrow \mathcal{P}_{c p, c}(\mathbb{R})$ is an $L^{1}$ Carathéodory multivalued map;

$\left(\mathrm{H}_{4}\right)$ there exists a function $q \in C([0,1], \mathbb{R})$ with $q(t)>0$ for a.e. $t \in[0,1]$ and a nondecreasing function $\psi: \mathbb{R}^{+} \rightarrow(0, \infty)$ such that

$$
\|G(t, x)\|:=\sup \{|v|: v \in G(t, x)\} \leq q(t) \psi(\|x\|) \text { a.e. } t \in[0,1],
$$

for all $x \in \mathbb{R}$;

$\left(\mathrm{H}_{5}\right)$ there exists a number $M>0$ such that

$$
\frac{\left(1-Z_{1}\|k\|\right) M}{Z_{1} F_{0}+Z_{2}\|q\| \psi(M)}>1
$$

where $Z_{1}, Z_{2}$ are given by (3.2) and (3.3), respectively, and $F_{0}=\int_{0}^{1}\|F(t, 0)\| d t$.

Then the problem (1.1) has a solution on $[0,1]$.

Proof To transform the problem (1.1) to a fixed-point problem, let us define an operator $\mathcal{N}: C([0,1], \mathbb{R}) \longrightarrow \mathcal{P}(C([0,1], \mathbb{R}))$ by

$$
\mathcal{N}(x)=\left\{\begin{array}{l}
h \in C([0,1], \mathbb{R}): \\
h(t)=\left\{\begin{array}{c}
-A \int_{0}^{t} \frac{(t-s)^{\alpha-1}}{\Gamma(\alpha)} f(s) d s-B \int_{0}^{t} \frac{(t-s)^{\alpha+\beta-1}}{\Gamma(\alpha+\beta)} g(s) d s \\
+Q t^{\alpha-1}\left[A \int_{0}^{1} \frac{(1-s)^{\alpha-\delta-1}}{\Gamma(\alpha-\delta)} f(s) d s\right. \\
+B \int_{0}^{1} \frac{(1-s)^{\alpha-\delta+\beta-1}}{\Gamma(\alpha-\delta+\beta)} g(s) d s-A \int_{0}^{\eta} \frac{(\eta-s)^{\alpha-\delta}}{\Gamma(\alpha-\delta+1)} f(s) d s \\
\left.-B \int_{0}^{\eta} \frac{(\eta-s)^{\alpha-\delta+\beta}}{\Gamma(\alpha-\delta+\beta+1)} g(s) d s\right]
\end{array}\right\}
\end{array}\right.
$$

for $f \in S_{F, x}, g \in S_{G, x}$, where $Q$ is given by (3.1).

We study the integral inclusion in the space $C([0,1], \mathbb{R})$ of all continuous real valued functions on $[0,1]$ with supremum norm $\|\cdot\|$. Define two multivalued maps $\mathcal{N}_{1}, \mathcal{N}_{2}$ : 


$$
\begin{aligned}
& C([0,1], \mathbb{R}) \rightarrow \mathcal{P}(C([0,1], \mathbb{R})) \text { by } \\
& \mathcal{N}_{1}(x)=\left\{\begin{array}{l}
h \in C([0,1], \mathbb{R}): \\
h(t)=\left\{\begin{array}{c}
-A \int_{0}^{t} \frac{(t-s){ }^{\alpha-1}}{\Gamma(\alpha)} f(s) d s \\
+A Q t^{\alpha-1}\left[\int_{0}^{1} \frac{(1-s)^{\alpha-\delta-1}}{\Gamma(\alpha-\delta)} f(s) d s\right. \\
\left.-A \int_{0}^{\eta} \frac{(\eta-s)^{\alpha-\delta}}{\Gamma(\alpha-\delta+1)} f(s) d s\right]
\end{array}\right\}
\end{array}\right.
\end{aligned}
$$

for $f \in S_{F, x}$ and

$$
\mathcal{N}_{2}(x)=\left\{\begin{array}{l}
h \in C([0,1], \mathbb{R}): \\
h(t)=\left\{\begin{array}{c}
-B \int_{0}^{t} \frac{(t-s)^{\alpha+\beta-1}}{\Gamma(\alpha+\beta)} g(s) d s \\
+B Q t^{\alpha-1}\left[\int_{0}^{1} \frac{(1-s)^{\alpha-\delta+\beta-1}}{\Gamma(\alpha-\delta+\beta)} g(s) d s\right. \\
\left.-B \int_{0}^{\eta} \frac{(\eta-s)^{\alpha-\delta+\beta}}{\Gamma(\alpha-\delta+\beta+1)} g(s) d s\right]
\end{array}\right\}
\end{array}\right.
$$

for $g \in S_{G, x}$.

Observe that $\mathcal{N}=\mathcal{N}_{1}+\mathcal{N}_{2}$. We shall show that the operators $\mathcal{N}_{1}$ and $\mathcal{N}_{2}$ satisfy all the conditions of Theorem 2.4 on $[0,1]$. For the sake of clarity, we split the proof into a sequence of steps and claims.

Step 1 . We show that $\mathcal{N}_{1}$ is a multivalued contraction on $C([0,1], \mathbb{R})$.

Let $x, y \in C([0,1], \mathbb{R})$ and $u_{1} \in \mathcal{N}_{1}(x)$. Then $u_{1} \in \mathcal{P}(C([0,1], \mathbb{R}))$ and

$$
\begin{aligned}
u_{1}(t)= & -A \int_{0}^{t} \frac{(t-s)^{\alpha-1}}{\Gamma(\alpha)} v_{1}(s) d s \\
& +A Q t^{\alpha-1}\left[\int_{0}^{1} \frac{(1-s)^{\alpha-\delta-1}}{\Gamma(\alpha-\delta)} v_{1}(s) d s-A \int_{0}^{\eta} \frac{(\eta-s)^{\alpha-\delta}}{\Gamma(\alpha-\delta+1)} v_{1}(s) d s\right],
\end{aligned}
$$

for some $v_{1} \in S_{F, x}$. Since $H(F(t, x), F(t, y)) \leq k(t)\|x-y\|$, there exists $w \in F(t, y)$ such that $\left|v_{1}(t)-w(t)\right| \leq k(t)\|x-y\|$. Thus the multivalued operator $U$ is defined by $U(t)=S_{F, y} \cap K(t)$, where

$$
K(t)=\left\{w \in \mathbb{R}|| v_{1}(t)-w(t) \mid \leq k(t)\|x-y\|\right\}
$$

has nonempty values and is measurable. Let $v_{2}$ be a measurable selection for $U$ (which exists by Kuratowski-Ryll-Nardzewski's selection theorem [21, 22]). Then $v_{2} \in F(t, y)$ and $\left|v_{1}(t)-v_{2}(t)\right| \leq k(t)\|x-y\|$ a.e. on $[0,1]$.

Define

$$
\begin{aligned}
u_{2}(t)= & -A \int_{0}^{t} \frac{(t-s)^{\alpha-1}}{\Gamma(\alpha)} v_{2}(s) d s \\
& +A Q t^{\alpha-1}\left[\int_{0}^{1} \frac{(1-s)^{\alpha-\delta-1}}{\Gamma(\alpha-\delta)} v_{2}(s) d s-\int_{0}^{\eta} \frac{(\eta-s)^{\alpha-\delta}}{\Gamma(\alpha-\delta+1)} v_{2}(s) d s\right] .
\end{aligned}
$$

It follows that $u_{2} \in \mathcal{N}_{1}(y)$ and

$$
\begin{aligned}
\left|u_{1}(t)-u_{2}(t)\right| \leq & \mid-A \int_{0}^{t} \frac{(t-s)^{\alpha-1}}{\Gamma(\alpha)}\left[v_{1}(s)-v_{2}(s)\right] d s \\
& +A Q t^{\alpha-1}\left[\int_{0}^{1} \frac{(1-s)^{\alpha-\delta-1}}{\Gamma(\alpha-\delta)}\left[v_{1}(s)-v_{2}(s)\right](s) d s\right.
\end{aligned}
$$




$$
\begin{aligned}
& \left.-\int_{0}^{\eta} \frac{(\eta-s)^{\alpha-\delta}}{\Gamma(\alpha-\delta+1)}\left[v_{1}(s)-v_{2}(s)\right](s) d s\right] \mid \\
\leq & |A| \int_{0}^{1} \frac{(1-s)^{\alpha-1}}{\Gamma(\alpha)}\left|v_{1}(s)-v_{2}(s)\right| d s \\
& +|A||Q| \int_{0}^{1} \frac{(1-s)^{\alpha-\delta-1}}{\Gamma(\alpha-\delta)}\left|v_{1}(s)-v_{2}(s)\right| d s \\
& +|A||Q| \int_{0}^{\eta} \frac{(\eta-s)^{\alpha-\delta}}{\Gamma(\alpha-\delta+1)}\left|v_{1}(s)-v_{2}(s)\right| d s \\
\leq & \left\{\frac{|A|}{\Gamma(\alpha+1)}+\frac{|A||Q|}{\Gamma(\alpha-\delta+1)}+\frac{|A \| Q| \eta^{\alpha-\delta+1}}{\Gamma(\alpha-\delta+2)}\right\}\|k\|\|x-y\| .
\end{aligned}
$$

Taking the supremum over the interval $[0,1]$, we obtain

$$
\left\|u_{1}-u_{2}\right\| \leq\left\{\frac{|A|}{\Gamma(\alpha+1)}+\frac{|A \| Q|}{\Gamma(\alpha-\delta+1)}+\frac{|A||Q| \eta^{\alpha-\delta+1}}{\Gamma(\alpha-\delta+2)}\right\}\|k\|\|x-y\|
$$

Combining the inequality (3.5) with the corresponding one obtained by interchanging the roles of $x$ and $y$, we get

$$
H\left(\mathcal{N}_{1}(x), \mathcal{N}_{1}(y)\right) \leq\left\{\frac{|A||Q|}{\Gamma(\alpha+1)}+\frac{|A||Q|}{\Gamma(\alpha-\delta+1)}+\frac{|A \| Q| \eta^{\alpha-\delta+1}}{\Gamma(\alpha-\delta+2)}\right\}\|k\|\|x-y\|,
$$

for all $x, y \in C([0,1], \mathbb{R})$. This shows that $\mathcal{N}_{1}$ is a multivalued contraction as

$$
Z_{1}\|k\|=\left\{\frac{|A|}{\Gamma(\alpha+1)}+\frac{|A \| Q|}{\Gamma(\alpha-\delta+1)}+\frac{|A \| Q| \eta^{\alpha-\delta+1}}{\Gamma(\alpha-\delta+2)}\right\}\|k\|<1 .
$$

Step 2. We shall show that the operator $\mathcal{N}_{2}$ is u.s.c. and compact. It is well known [23, Proposition 1.2] that if an operator is completely continuous and has a closed graph, then it is u.s.c. Therefore we will prove that $\mathcal{N}_{2}$ is completely continuous and has a closed graph. This step involves several claims.

Claim I $\mathcal{N}_{2}$ maps bounded sets into bounded sets in $C([0,1], \mathbb{R})$.

Let $B_{r}=\{x \in C([0,1], \mathbb{R}):\|x\| \leq r\}$ be a bounded set in $C([0,1], \mathbb{R})$.

Now for each $u \in \mathcal{N}_{2}(x)$, there exists a $w \in S_{G, x}$ such that

$$
\begin{aligned}
u(t)= & -B \int_{0}^{t} \frac{(t-s)^{\alpha+\beta-1}}{\Gamma(\alpha+\beta)} w(s) d s \\
& +B Q t^{\alpha-1}\left[\int_{0}^{1} \frac{(1-s)^{\alpha-\delta+\beta-1}}{\Gamma(\alpha-\delta+\beta)} w(s) d s-\int_{0}^{\eta} \frac{(\eta-s)^{\alpha-\delta+\beta}}{\Gamma(\alpha-\delta+\beta+1)} w(s) d s\right] .
\end{aligned}
$$

Then for each $t \in[0,1]$,

$$
\begin{aligned}
|u(t)| \leq & |B| \int_{0}^{t} \frac{(t-s)^{\alpha+\beta-1}}{\Gamma(\alpha+\beta)}|w(s)| d s \\
& +|B||Q|\left[\int_{0}^{1} \frac{(1-s)^{\alpha-\delta+\beta-1}}{\Gamma(\alpha-\delta+\beta)}|w(s)| d s+\int_{0}^{\eta} \frac{(\eta-s)^{\alpha-\delta+\beta}}{\Gamma(\alpha-\delta+\beta+1)}|w(s)| d s\right]
\end{aligned}
$$




$$
\begin{aligned}
\leq & |B| \int_{0}^{1} \frac{(1-s)^{\alpha+\beta-1}}{\Gamma(\alpha+\beta)} q(t) \psi(\|x\|) d s \\
& +|B \| Q|\left[\int_{0}^{1} \frac{(1-s)^{\alpha-\delta+\beta-1}}{\Gamma(\alpha-\delta+\beta)} q(t) \psi(\|x\|) d s\right. \\
& \left.+\int_{0}^{\eta} \frac{(\eta-s)^{\alpha-\delta+\beta}}{\Gamma(\alpha-\delta+\beta+1)} q(t) \psi(\|x\|) d s\right] \\
\leq & \psi(\|x\|)\|q\|\left\{\frac{|B|}{\Gamma(\alpha+\beta+1)}+\frac{|B \| Q|}{\Gamma(\alpha-\delta+\beta+1)}+\frac{|B \| Q| \eta^{\alpha-\delta+\beta+1}}{\Gamma(\alpha-\delta+\beta+2)}\right\},
\end{aligned}
$$

which implies that

$$
\|u\| \leq \psi(r)\|q\|\left\{\frac{|B|}{\Gamma(\alpha+\beta+1)}+\frac{|B||Q|}{\Gamma(\alpha-\delta+\beta+1)}+\frac{|B||Q| \eta^{\alpha-\delta+\beta+1}}{\Gamma(\alpha-\delta+\beta+2)}\right\} .
$$

Hence $\mathcal{N}_{2}$ is bounded.

Claim II $\mathcal{N}_{2}$ maps bounded sets into equicontinuous sets.

As in the proof of Claim I, let $B_{r}$ be a bounded set and $u \in \mathcal{N}_{2}(x)$ for some $x \in B_{r}$. Then there exists $w \in S_{G, x}$ such that

$$
\begin{aligned}
u(t)= & -B \int_{0}^{t} \frac{(t-s)^{\alpha+\beta-1}}{\Gamma(\alpha+\beta)} w(s) d s \\
& +B Q t^{\alpha-1}\left[\int_{0}^{1} \frac{(1-s)^{\alpha-\delta+\beta-1}}{\Gamma(\alpha-\delta+\beta)} w(s) d s-\int_{0}^{\eta} \frac{(\eta-s)^{\alpha-\delta+\beta}}{\Gamma(\alpha-\delta+\beta+1)} w(s) d s\right] .
\end{aligned}
$$

Then for any $t_{1}, t_{2} \in[0,1]$ with $t_{1} \leq t_{2}$ we have

$$
\begin{aligned}
& \left|u\left(t_{1}\right)-u\left(t_{2}\right)\right| \\
& \leq|B| \int_{0}^{t_{2}} \frac{\left[\left(t_{2}-s\right)^{\alpha+\beta-1}-\left(t_{1}-s\right)^{\alpha+\beta-1}\right]}{\Gamma(\alpha+\beta)}|w(s)| d s+|B| \int_{t_{1}}^{t_{2}} \frac{\left(t_{2}-s\right)^{\alpha+\beta-1}}{\Gamma(\alpha+\beta)}|w(s)| d s \\
& \quad+|B||Q|\left|t_{2}^{\alpha-1}-t_{1}^{\alpha-1}\right|\left[\int_{0}^{1} \frac{(1-s)^{\alpha-\delta+\beta-1}}{\Gamma(\alpha-\delta+\beta)}|w(s)| d s+\int_{0}^{\eta} \frac{(\eta-s)^{\alpha-\delta+\beta}}{\Gamma(\alpha-\delta+\beta+1)}|w(s)| d s\right] \\
& \leq|B| \int_{0}^{t_{2}} \frac{\left[\left(t_{2}-s\right)^{\alpha+\beta-1}-\left(t_{1}-s\right)^{\alpha+\beta-1}\right]}{\Gamma(\alpha+\beta)} q(s) \psi(r) d s \\
& \quad+|B| \int_{t_{1}}^{t_{2}} \frac{\left(t_{2}-s\right)^{\alpha+\beta-1}}{\Gamma(\alpha+\beta)} q(s) \psi(r) d s \\
& \quad+|B||Q|\left|t_{2}^{\alpha-1}-t_{1}^{\alpha-1}\right|\left[\int_{0}^{1} \frac{(1-s)^{\alpha-\delta+\beta-1}}{\Gamma(\alpha-\delta+\beta)} q(s) \psi(r) d s\right. \\
& \left.\quad+\int_{0}^{\eta} \frac{(\eta-s)^{\alpha-\delta+\beta}}{\Gamma(\alpha-\delta+\beta+1)} q(s) \psi(r) d s\right] .
\end{aligned}
$$

Obviously the right hand side of the above inequality tends to zero independently of $x \in B_{r}$ as $t_{1}-t_{2} \rightarrow 0$. Therefore it follows by the Arzelá-Ascoli theorem that $\mathcal{N}_{2}: C([0,1], \mathbb{R}) \rightarrow$ $\mathcal{P}(C([0,1], \mathbb{R}))$ is completely continuous. 
Claim III Next we prove that $\mathcal{N}_{2}$ has a closed graph.

Let $x_{n} \rightarrow x_{*}, h_{n} \in \mathcal{N}_{2}\left(x_{n}\right)$ and $h_{n} \rightarrow h_{*}$. Then we need to show that $h_{*} \in \mathcal{B}\left(x_{*}\right)$. Associated with $h_{n} \in \mathcal{B}\left(x_{n}\right)$, there exists $v_{n} \in S_{G, x_{n}}$ such that for each $t \in[0,1]$,

$$
\begin{aligned}
h_{n}(t)= & -B \int_{0}^{t} \frac{(t-s)^{\alpha+\beta-1}}{\Gamma(\alpha+\beta)} v_{n}(s) d s \\
& +B Q t^{\alpha-1}\left[\int_{0}^{1} \frac{(1-s)^{\alpha-\delta+\beta-1}}{\Gamma(\alpha-\delta+\beta)} v_{n}(s) d s-\int_{0}^{\eta} \frac{(\eta-s)^{\alpha-\delta+\beta}}{\Gamma(\alpha-\delta+\beta+1)} v_{n}(s) d s\right] .
\end{aligned}
$$

Thus it suffices to show that there exists $v_{*} \in S_{G, x_{*}}$ such that for each $t \in[0,1]$,

$$
\begin{aligned}
h_{*}(t)= & -B \int_{0}^{t} \frac{(t-s)^{\alpha+\beta-1}}{\Gamma(\alpha+\beta)} v_{*}(s) d s \\
& +B Q t^{\alpha-1}\left[\int_{0}^{1} \frac{(1-s)^{\alpha-\delta+\beta-1}}{\Gamma(\alpha-\delta+\beta)} v_{*}(s) d s-\int_{0}^{\eta} \frac{(\eta-s)^{\alpha-\delta+\beta}}{\Gamma(\alpha-\delta+\beta+1)} v(s) d s\right] .
\end{aligned}
$$

Let us consider the linear operator $\Theta: L^{1}([0,1], \mathbb{R}) \rightarrow C([0,1], \mathbb{R})$ given by

$$
\begin{aligned}
v \mapsto \Theta(v)(t)= & -B \int_{0}^{t} \frac{(t-s)^{\alpha+\beta-1}}{\Gamma(\alpha+\beta)} v(s) d s \\
& +B Q t^{\alpha-1}\left[\int_{0}^{1} \frac{(1-s)^{\alpha-\delta+\beta-1}}{\Gamma(\alpha-\delta+\beta)} v(s) d s-\int_{0}^{\eta} \frac{(\eta-s)^{\alpha-\delta+\beta}}{\Gamma(\alpha-\delta+\beta+1)} v(s) d s\right] .
\end{aligned}
$$

Observe that

$$
\begin{aligned}
\left\|h_{n}(t)-h_{*}(t)\right\|= & \|-B \int_{0}^{t} \frac{(t-s)^{\alpha+\beta-1}}{\Gamma(\alpha+\beta)}\left(v_{n}(u)-v_{*}(u)\right) d s \\
& +B Q t^{\alpha-1}\left[\int_{0}^{1} \frac{(1-s)^{\alpha-\delta+\beta-1}}{\Gamma(\alpha-\delta+\beta)}\left(v_{n}(u)-v_{*}(u)\right) d s\right. \\
& \left.-\int_{0}^{\eta} \frac{(\eta-s)^{\alpha-\delta+\beta}}{\Gamma(\alpha-\delta+\beta+1)}\left(v_{n}(u)-v_{*}(u)\right) d s\right] \| \rightarrow 0,
\end{aligned}
$$

as $n \rightarrow \infty$. Thus, it follows by Lemma 2.6 that $\Theta \circ S_{G}$ is a closed graph operator. Further, we have $h_{n}(t) \in \Theta\left(S_{G, x_{n}}\right)$. Since $x_{n} \rightarrow x_{*}$, we have

$$
\begin{aligned}
h_{*}(t)= & -B \int_{0}^{t} \frac{(t-s)^{\alpha+\beta-1}}{\Gamma(\alpha+\beta)} v_{*}(s) d s \\
& +B Q t^{\alpha-1}\left[\int_{0}^{1} \frac{(1-s)^{\alpha-\delta+\beta-1}}{\Gamma(\alpha-\delta+\beta)} v_{*}(s) d s-\int_{0}^{\eta} \frac{(\eta-s)^{\alpha-\delta+\beta}}{\Gamma(\alpha-\delta+\beta+1)} v(s) d s\right]
\end{aligned}
$$

for some $v_{*} \in S_{G, x_{*}}$.

Hence $\mathcal{N}_{2}$ has a closed graph (and therefore it has closed values). In consequence, $\mathcal{N}_{2}$ is compact valued.

Therefore the operators $\mathcal{N}_{1}$ and $\mathcal{N}_{2}$ satisfy all the conditions of Theorem 2.4. So the conclusion of Theorem 2.4 applies and either condition (i) or condition (ii) holds. We show that the conclusion (ii) is not possible. If $x \in \lambda \mathcal{N}_{1}(x)+\lambda \mathcal{N}_{2}(x)$ for $\lambda \in(0,1)$, then 
there exist $v_{1} \in S_{F, x}$ and $v_{2} \in S_{G, x}$ such that

$$
\begin{aligned}
x(t)= & \lambda\left\{-A \int_{0}^{t} \frac{(t-s)^{\alpha-1}}{\Gamma(\alpha)} v_{1}(s) d s\right. \\
& \left.+Q A t^{\alpha-1}\left[\int_{0}^{1} \frac{(1-s)^{\alpha-\delta-1}}{\Gamma(\alpha-\delta)} v_{1}(s) d s-\int_{0}^{\eta} \frac{(\eta-s)^{\alpha-\delta}}{\Gamma(\alpha-\delta+1)} v_{1}(s) d s\right]\right\} \\
& +\lambda^{-1}\left\{-B \int_{0}^{t} \frac{(t-s)^{\alpha+\beta-1}}{\Gamma(\alpha+\beta)} v_{2}(s) d s\right. \\
& +Q B t^{\alpha-1}\left[\int_{0}^{1} \frac{(1-s)^{\alpha-\delta+\beta-1}}{\Gamma(\alpha-\delta+\beta)} v_{2}(s) d s\right. \\
& \left.\left.-\int_{0}^{\eta} \frac{(\eta-s)^{\alpha-\delta+\beta}}{\Gamma(\alpha-\delta+\beta+1)} v_{2}(s) d s\right]\right\} .
\end{aligned}
$$

By hypothesis $\left(\mathrm{H}_{2}\right)$, for all $t \in[0,1]$, we have

$$
\begin{aligned}
\|F(t, x)\| & =H(F(t, x), 0) \leq H(F(t, x), F(t, 0))+H(F(t, 0), 0) \\
& \leq H(F(t, x), F(t, 0))+\|F(t, 0)\| .
\end{aligned}
$$

Hence for any $a \in F(t, x)$,

$$
\begin{aligned}
|a| & \leq\|F(t, x)\| \leq H(F(t, x), F(t, 0))+\|F(t, 0)\| \\
& \leq k(t)\|x\|+\|F(t, 0)\|,
\end{aligned}
$$

for all $t \in[0,1]$. Then we have

$$
\begin{aligned}
|x(t)| \leq & |A|\left\{\int_{0}^{t} \frac{(t-s)^{\alpha-1}}{\Gamma(\alpha)}[k(s)\|x\|+\|F(t, 0)\|] d s\right. \\
& +|Q \|| A \mid\left[\int_{0}^{1} \frac{(1-s)^{\alpha-\delta-1}}{\Gamma(\alpha-\delta)}[k(s)\|x\|+\|F(t, 0)\|] d s\right. \\
& \left.\left.-\int_{0}^{\eta} \frac{(\eta-s)^{\alpha-\delta}}{\Gamma(\alpha-\delta+1)}[k(s)\|x\|+\|F(t, 0)\|] d s\right]\right\} \\
& +|B|\left\{\int_{0}^{t} \frac{(t-s)^{\alpha+\beta-1}}{\Gamma(\alpha+\beta)} q(s) \psi(\|x\|) d s\right. \\
& +|Q \|| B \mid\left[\int_{0}^{1} \frac{(1-s)^{\alpha-\delta+\beta-1}}{\Gamma(\alpha-\delta+\beta)} q(s) \psi(\|x\|) d s\right. \\
& \left.\left.-\int_{0}^{\eta} \frac{(\eta-s)^{\alpha-\delta+\beta}}{\Gamma(\alpha-\delta+\beta+1)} q(s) \psi(\|x\|) d s\right]\right\} \\
\leq & \left\{\frac{|A|}{\Gamma(\alpha+1)}+\frac{|A||Q|}{\Gamma(\alpha-\delta+1)}+\frac{|A \| Q| \eta^{\alpha-\delta+1}}{\Gamma(\alpha-\delta+2)}\right\}\left(\|k\|\|x\|+F_{0}\right) \\
& +\left\{\frac{|B|}{\Gamma(\alpha+\beta+1)}+\frac{|B \| Q|}{\Gamma(\alpha-\delta+\beta+1)}+\frac{|B \| Q| \eta^{\alpha-\delta+\beta+1}}{\Gamma(\alpha-\delta+\beta+2)}\right\}\|q\| \psi(\|x\|) \\
= & Z_{1}\left(\|k\|\|x\|+F_{0}\right)+Z_{2}\|q\| \psi(\|x\|) .
\end{aligned}
$$


Thus,

$$
\|x\| \leq Z_{1}\left(\|k\|\|x\|+F_{0}\right)+Z_{2}\|q\| \psi(\|x\|) .
$$

Now, if condition (ii) of Theorem 2.4 holds, then there exist $\lambda \in(0,1)$ and $x \in \partial B_{r}$ such that $x=\lambda \mathcal{N}(x)$. Then $x$ is a solution of (3.6) with $\|x\|=M$ and consequently, the inequality (3.7) yields

$$
\frac{\left(1-Z_{1}\|k\|\right) M}{Z_{1} F_{0}+Z_{2}\|q\| \psi(M)} \leq 1
$$

which contradicts (3.4). Hence, $\mathcal{N}$ has a fixed point in $[0,1]$ by Theorem 2.4 , which in fact is a solution of the problem (1.1). This completes the proof.

\subsection{The lower semi-continuous case}

This section is devoted to the study of the case that the maps in (1.1) are not necessarily convex-valued. We establish the existence result for the problem at hand by applying the nonlinear alternative of Leray-Schauder type and a selection theorem due to Bressan and Colombo [24] for lower semi-continuous maps with decomposable values. Before presenting this result, we revisit some basic concepts.

Let $\mathfrak{X}$ be a nonempty closed subset of a Banach space $\mathfrak{E}$ and $\mathfrak{G}: \mathfrak{X} \rightarrow \mathcal{P}(\mathfrak{E})$ be a multivalued operator with nonempty closed values. $\mathfrak{G}$ is lower semi-continuous (l.s.c.) if the set $\{y \in \mathfrak{X}: \mathfrak{G}(y) \cap \mathfrak{B} \neq \emptyset\}$ is open for any open set $\mathfrak{B}$ in $\mathfrak{E}$. Let $\mathfrak{M}$ be a subset of $[0,1] \times \mathbb{R}$. $\mathfrak{M}$ is $\mathcal{L} \otimes \mathfrak{B}$ measurable if $\mathfrak{M}$ belongs to the $\sigma$ algebra generated by all sets of the form $\mathcal{J} \times \mathcal{D}$, where $\mathcal{J}$ is Lebesgue measurable in $[0,1]$ and $\mathcal{D}$ is Borel measurable in $\mathbb{R}$. A subset $\mathcal{S}$ of $L^{1}([0,1], \mathbb{R})$ is decomposable if for all $u, v \in \mathcal{S}$ and measurable $\mathcal{J} \subset[0,1]=J$, the function $u \chi_{\mathcal{J}}+v \chi_{\mathcal{J}-\mathcal{J}} \in \mathcal{S}$, where $\chi_{\mathcal{J}}$ stands for the characteristic function of $\mathcal{J}$.

Definition 3.3 Let $Y$ be a separable metric space and let $\mathcal{W}: Y \rightarrow \mathcal{P}\left(L^{1}([0,1], \mathbb{R})\right)$ be a multivalued operator. We say $\mathcal{W}$ has a property $(\mathrm{BC})$ if $\mathcal{W}$ is lower semi-continuous (l.s.c.) and has nonempty closed and decomposable values.

Let $F:[0,1] \times \mathbb{R} \rightarrow \mathcal{P}(\mathbb{R})$ be a multivalued map with nonempty compact values. Define a multivalued operator $\mathcal{F}: C([0,1] \times \mathbb{R}) \rightarrow \mathcal{P}\left(L^{1}([0,1], \mathbb{R})\right)$ associated with $F$ as

$$
\mathcal{F}(x)=\left\{v \in L^{1}([0,1], \mathbb{R}): v(t) \in F(t, x(t)) \text { for a.e. } t \in[0,1]\right\},
$$

which is called the Nemytskii operator associated with $F$.

Definition 3.4 Let $F:[0,1] \times \mathbb{R} \rightarrow \mathcal{P}(\mathbb{R})$ be a multivalued function with nonempty compact values. We say $F$ is of lower semi-continuous type (l.s.c. type) if its associated $\mathrm{Ne}$ mytskii operator $\mathcal{F}$ is lower semi-continuous and has nonempty closed and decomposable values.

Lemma 3.5 ([25]) Let $Y$ be a separable metric space and let $\mathcal{W}: Y \rightarrow \mathcal{P}\left(L^{1}([0,1], \mathbb{R})\right)$ be a multivalued operator satisfying the property $(B C)$. Then $\mathcal{W}$ has a continuous selection, that is, there exists a continuous function (single-valued) $w: Y \rightarrow L^{1}([0,1], \mathbb{R})$ such that $w(x) \in \mathcal{W}(x)$ for every $x \in Y$. 
Theorem 3.6 Assume that $\left(\mathrm{H}_{2}\right),\left(\mathrm{H}_{4}\right),\left(\mathrm{H}_{5}\right)$, and the following condition hold:

$\left(\mathrm{H}_{6}\right) \quad F, G:[0,1] \times \mathbb{R} \rightarrow \mathcal{P}(\mathbb{R})$ are nonempty compact-valued multivalued maps such that

(a) $(t, x) \longmapsto F(t, x),(t, x) \longmapsto G(t, x)$ are $\mathcal{L} \otimes \mathcal{B}$ measurable,

(b) $x \longmapsto F(t, x), x \longmapsto G(t, x)$ are lower semicontinuous for each $t \in[0,1]$;

Then the boundary value problem (1.1) has at least one solution on $[0,1]$.

Proof It follows from $\left(\mathrm{H}_{2}\right),\left(\mathrm{H}_{4}\right)$, and $\left(\mathrm{H}_{6}\right)$ that $F$ and $G$ are of 1.s.c. type. Then from Lemma 3.5, there exist continuous functions $f, g: C([0,1], \mathbb{R}) \rightarrow L^{1}([0,1], \mathbb{R})$ such that $f(x) \in \mathcal{F}(x), g(x) \in \mathcal{G}(x)$ for all $x \in C([0,1], \mathbb{R})$.

Consider the problem

$$
\left\{\begin{array}{l}
-D^{\alpha} x(t)=A f(x(t))+B I^{\beta} g(x(t)), \quad 2<\alpha \leq 3, t \in[0,1], \\
D^{\delta} x(0)=D^{\delta+1} x(0)=0, \quad D^{\delta} x(1)=\int_{0}^{\eta} D^{\delta} x(s) d s, \quad 0<\eta<1 .
\end{array}\right.
$$

Observe that if $x \in A C^{2}([0,1])$ is a solution of (3.8), then $x$ is a solution to the problem (1.1). Now, we define two multivalued operators $\widetilde{\mathcal{N}}_{1}, \widetilde{\mathcal{N}}_{2}: C([0,1], \mathbb{R}) \longrightarrow \mathcal{P}(C([0,1], \mathbb{R}))$ by

$$
\tilde{\mathcal{N}}_{1} x(t)=\left\{\begin{array}{c}
-A \int_{0}^{t} \frac{(t-s)^{\alpha-1}}{\Gamma(\alpha)} f(x(s)) d s \\
+A Q t^{\alpha-1}\left[\int_{0}^{1} \frac{(1-s)^{\alpha-\delta-1}}{\Gamma(\alpha-\delta)} f(x(s)) d s\right. \\
\left.-A \int_{0}^{\eta} \frac{(\eta-s)^{\alpha-\delta}}{\Gamma(\alpha-\delta+1)} f(x(s)) d s\right]
\end{array}\right.
$$

and

$$
\tilde{\mathcal{N}}_{2} x(t)=\left\{\begin{array}{c}
-B \int_{0}^{t} \frac{(t-s)^{\alpha+\beta-1}}{\Gamma(\alpha+\beta)} g(x(s)) d s \\
+B Q t^{\alpha-1}\left[\int_{0}^{1} \frac{(1-s)^{\alpha-\delta+\beta-1}}{\Gamma(\alpha-\delta+\beta)} g(x(s)) d s\right. \\
\left.-B \int_{0}^{\eta} \frac{(\eta-s)^{\alpha-\delta+\beta}}{\Gamma(\alpha-\delta+\beta+1)} g(x(s)) d s\right] .
\end{array}\right.
$$

Clearly $\widetilde{\mathcal{N}}_{1}, \widetilde{\mathcal{N}}_{2}: C([0,1], \mathbb{R}) \rightarrow C([0,1], \mathbb{R})$ are continuous. Also the argument in Theorem 3.2 guarantees that $\widetilde{\mathcal{N}}_{1}$ and $\widetilde{\mathcal{N}}_{2}$ satisfy all the conditions of the nonlinear alternative for contractive maps in the single-valued setting [26] and hence the problem (3.8) has a solution.

Example 3.7 Consider the following fractional boundary value problem:

$$
\left\{\begin{array}{l}
-D^{5 / 2} x(t) \in 3 F(t, x(t))+I^{3 / 4} G(t, x(t)), \quad t \in[0,1], \\
D^{1 / 8} x(0)=D^{9 / 8} x(0)=0, \quad D^{1 / 8} x(1)=\int_{0}^{1 / 2} D^{1 / 8} x(s) d s,
\end{array}\right.
$$

where

$$
F(t, x)=\left[-\frac{18}{(3+t)^{2}}-\frac{\sin x}{(4+t)^{2}}-2,-\frac{1}{10}\right], \quad G(t, x)=\left[\frac{|x|^{3}}{8\left(|x|^{3}+3\right)}, \frac{|x|}{9(|x|+1)}\right] .
$$

We have

$$
\sup \{|u|: u \in F(t, x)\} \leq 2+\frac{1}{(4+t)^{2}}+\frac{18}{(3+t)^{2}}, \quad H(F(t, x), F(t, \bar{x})) \leq k(t)|x-\bar{x}|
$$


with $k(t)=\frac{1}{(4+t)^{2}}$. Using the given data, we find that

$$
\begin{aligned}
& Q=\frac{\Gamma(\alpha-\delta+1)}{\left(\alpha-\delta-\eta^{\alpha-\delta}\right) \Gamma(\alpha)} \simeq 1.000669, \quad\|k\|=1 / 20, \\
& \|q\|=1 / 8, \quad \psi(M)=1, \quad F_{0}=3.5, \\
& Z_{1}=\frac{|A|}{\Gamma(\alpha+1)}+\frac{|A||Q|}{\Gamma(\alpha-\delta+1)}+\frac{|A \| Q| \eta^{\alpha-\delta+1}}{\Gamma(\alpha-\delta+2)} \simeq 1.031919, \\
& Z_{2}=\frac{|B|}{\Gamma(\alpha+\beta+1)}+\frac{|B||Q|}{\Gamma(\alpha-\delta+\beta+1)}+\frac{|B||Q| \eta^{\alpha-\delta+\beta+1}}{\Gamma(\alpha-\delta+\beta+2)} \simeq 0.264907 .
\end{aligned}
$$

Clearly $Z_{1}\|k\|<1$, and by the condition:

$$
\frac{\left(1-Z_{1}\|k\|\right) M}{Z_{1} F_{0}+Z_{2}\|q\| \psi(M)}>1
$$

it is found that $M>M_{1}$, where $M_{1} \simeq 3.843119$. Thus, all the assumptions of Theorem 3.2 are satisfied. Hence, the conclusion of Theorem 3.2 applies to the problem (3.9).

\section{Competing interests}

The authors declare that they have no competing interests.

\section{Authors' contributions}

Each of the authors, AA, SKN, BA and HHA, contributed to each part of this work equally and read and approved the final version of the manuscript.

\section{Author details}

${ }^{1}$ Department of Mathematics, Faculty of Science, King Abdulaziz University, P.O. Box 80203, Jeddah, 21589, Saudi Arabia.

${ }^{2}$ Department of Mathematics, University of loannina, loannina, 451 10, Greece.

\section{Authors' information}

Member of Nonlinear Analysis and Applied Mathematics (NAAM)-Research Group at King Abdulaziz University, Jeddah, Saudi Arabia.

\section{Acknowledgements}

This project was funded by the Deanship of Scientific Research (DSR), King Abdulaziz University under grant no. 3-130/1433/HiCi. The authors, therefore, acknowledge with thanks DSR technical and financial support.

Received: 18 November 2013 Accepted: 10 January 2014 Published: 30 Jan 2014

\section{References}

1. Samko, SG, Kilbas, AA, Marichev, Ol: Fractional Integrals and Derivatives, Theory and Applications. Gordon and Breach, Yverdon (1993)

2. Podlubny, I: Fractional Differential Equations. Academic Press, San Diego (1999)

3. Kilbas, AA, Srivastava, HM, Trujillo, JJ: Theory and Applications of Fractional Differential Equations. North-Holland Mathematics Studies, vol. 204. Elsevier, Amsterdam (2006)

4. Baleanu, D, Diethelm, K, Scalas, E, Trujillo, JJ: Fractional Calculus Models and Numerical Methods. Series on Complexity, Nonlinearity and Chaos. World Scientific, Boston (2012)

5. Henderson, J, Ouahab, A: Fractional functional differential inclusions with finite delay. Nonlinear Anal. 70, 2091-2105 (2009)

6. Chang, Y-K, Nieto, JJ: Some new existence results for fractional differential inclusions with boundary conditions Math. Comput. Model. 49, 605-609 (2009)

7. Benchohra, M, Hamani, S, Ntouyas, SK: Boundary value problems for differential equations with fractional order and nonlocal conditions. Nonlinear Anal. 71, 2391-2396 (2009)

8. Cernea, A: On the existence of solutions for nonconvex fractional hyperbolic differential inclusions. Commun. Math Anal. 9(1), 109-120 (2010)

9. Agarwal, RP, Benchohra, M, Hamani, S: A survey on existence results for boundary value problems of nonlinear fractional differential equations and inclusions. Acta Appl. Math. 109, 973-1033 (2010)

10. Ahmad, B, Ntouyas, SK: Some existence results for boundary value problems of fractional differential inclusions with non-separated boundary conditions. Electron. J. Qual. Theory Differ. Equ. 2010, 71 (2010)

11. Ahmad, B, Ntouyas, SK, Alsaedi, A: New existence results for nonlinear fractional differential equations with three-point integral boundary conditions. Adv. Differ. Equ. 2011, Article ID 107384 (2011) 
12. Baleanu, D, Mustafa, OG, O'Regan, D: A Nagumo-like uniqueness theorem for fractional differential equations. J. Phys. A, Math. Theor. 44(39), Article ID 392003 (2011)

13. Ahmad, B, Ntouyas, SK: Nonlinear fractional differential equations and inclusions of arbitrary order and multi-strip boundary conditions. Electron. J. Differ. Equ. 2012, 98 (2012)

14. Ahmad, B, Nieto, J: Sequential fractional differential equations with three-point boundary conditions. Comput. Math. Appl. 64, 3046-3052 (2012)

15. Sudsutad, W, Tariboon, J: Existence results of fractional integro-differential equations with $m$-point multi-term fractional order integral boundary conditions. Bound. Value Probl. 2012, 94 (2012)

16. Wang, G, Ahmad, B, Zhang, L, Agarwal, RP: Nonlinear fractional integro-differential equations on unbounded domains in a Banach space. J. Comput. Appl. Math. 249, 51-56 (2013)

17. Babakhani, A, Baleanu, D, Agarwal, RP: The existence and uniqueness of solutions for a class of nonlinear fractional differential equations with infinite delay. Abstr. Appl. Anal. 2013, Art. ID 592964 (2013)

18. Ntouyas, SK: Boundary value problems for nonlinear fractional differential equations and inclusions with nonlocal and fractional integral boundary conditions. Opusc. Math. 33, 117-138 (2013)

19. Petryshyn, WV, Fitzpatric, PM: A degree theory, fixed point theorems, and mapping theorems for multivalued noncompact maps. Trans. Am. Math. Soc. 194, 1-25 (1974)

20. Lasota, A, Opial, Z: An application of the Kakutani-Ky Fan theorem in the theory of ordinary differential equations. Bull. Acad. Pol. Sci., Sér. Sci. Math. Astron. Phys. 13, 781-786 (1965)

21. Kuratowski, K, Ryll-Nardzewski, C: A general theorem on selectors. Bull. Acad. Pol. Sci., Sér. Sci. Math. Astron. Phys. 13, 397-403 (1965)

22. Gorniewicz, L: Topological Fixed Point Theory of Multivalued Mappings. Springer, Dordrecht (2006)

23. Deimling, K: Multivalued Differential Equations. de Gruyter, Berlin (1992)

24. Bressan, A, Colombo, G: Extensions and selections of maps with decomposable values. Stud. Math. 90, 69-86 (1988)

25. Frigon, M: Théorèmes d'existence de solutions d'inclusions différentielles. In: Granas, A, Frigon, M (eds.) Topological Methods in Differential Equations and Inclusions. NATO ASI Series C, vol. 472, pp. 51-87. Kluwer Academic, Dordrecht (1995)

26. Granas, A, Dugundji, J: Fixed Point Theory. Springer, New York (2005)

$10.1186 / 1687-2770-2014-25$

Cite this article as: Alsaedi et al.: Existence results for fractional integral inclusions via nonlinear alternative for contractive maps. Boundary Value Problems 2014, 2014:25

\section{Submit your manuscript to a SpringerOpen ${ }^{\circ}$ journal and benefit from:}

- Convenient online submission

- Rigorous peer review

- Immediate publication on acceptance

- Open access: articles freely available online

- High visibility within the field

- Retaining the copyright to your article 